\title{
Boosted jet techniques for a supersymmetric scenario with gravitino LSP
}

\author{
Akanksha Bhardwaj, ${ }^{a, b}$ Juhi Dutta, ${ }^{c}$ Partha Konar, ${ }^{a}$ Biswarup Mukhopadhyaya ${ }^{d}$ \\ and Santosh Kumar Rai ${ }^{e}$ \\ ${ }^{a}$ Physical Research Laboratory, \\ Ahmedabad 380009, Gujarat, India \\ ${ }^{b}$ Indian Institute of Technology, \\ Gandhinagar 382424, Gujarat, India \\ ${ }^{c}$ Institut für Theoretische Physik, \\ Universität Hamburg, Luruper Chaussee 149, 22761 Hamburg, Germany \\ ${ }^{d}$ Department of Physical Sciences, \\ Indian Institute of Science Education and Research Kolkata, \\ Mohanpur, 741246, India \\ ${ }^{e}$ Regional Centre for Accelerator-based Particle Physics, \\ Harish-Chandra Research Institute, HBNI, \\ Chhatnag Road, Jhusi, Prayagraj 211019, India \\ E-mail: akanksha@prl.res.in, juhi.dutta@desy.de, konar@prl.res.in, \\ biswarup@iiserkol.ac.in, skrai@hri.res.in
}

ABSTRACT: Search for compressed supersymmetry at multi-TeV scale, in the presence of a light gravitino dark matter, can get sizable uplift while looking into the associated fatjets with missing transverse momenta as a signature of the boson produced in the decay process of much heavier next-to-lightest sparticle. We focus on the hadronic decay of the ensuing Higgs and/or $Z$ boson giving rise to at least two fat-jets and $\mathbb{E}_{T}$ in the final state. We perform a detailed background study adopting a multivariate analysis using a boosted decision tree to provide a robust investigation to explore the discovery potential for such signal at $14 \mathrm{TeV}$ LHC considering different benchmark points satisfying all the theoretical and experimental constraints. This channel provides the best discovery prospects with most of the benchmarks discoverable within an integrated luminosity of $\mathcal{L}=200 \mathrm{fb}^{-1}$. Kinematic observables are investigated in order to distinguish between compressed and uncompressed spectra having similar event yields.

KEYwords: Jets, Supersymmetry Phenomenology

ARXIV EPRINT: 2007.00351 


\section{Contents}

1 Introduction 1

2 Decay properties of a higgsino-like NLSP 3

3 Benchmarks $\quad 4$

4 Collider analysis $\quad 7$

$\begin{array}{lll}4.1 & \text { Signal topology } & 7\end{array}$

$\begin{array}{lll}4.2 & \text { Backgrounds } & 7\end{array}$

$\begin{array}{llr}4.3 & \text { Simulated events and data sample } & 8\end{array}$

4.4 Multivariate analysis 9

4.5 Complementary signals at high energy and high luminosity upgrades of LHC at $\sqrt{s}=27 \mathrm{TeV} \quad 13$

5 Distinction of compressed and uncompressed spectra $\quad 15$

$\begin{array}{lll}6 & \text { Summary and conclusions } & 17\end{array}$

\section{Introduction}

Improved analysis techniques, especially in the context of the high-luminosity Large Hadron Collider (LHC), are highly desirable in the pursuit of new fundamental physics. Supersymmetry (SUSY) has been one of the front-runner candidates for beyond standard model (BSM) physics for the last few decades, and its search at experiments provides common ground to many non-SUSY searches too. In view of the null results at the Run 1 and Run 2 of LHC, compressed SUSY (cSUSY) [1-4] has gained relevance in its ongoing pursuit, primarily aimed at looking at the elusive scenario of new physics with a significantly degenerate mass spectra. In such scenarios and more specifically in the minimal supersymmetric standard model (MSSM) with the lightest neutralino $\left(\widetilde{\chi}_{1}^{0}\right)$ as the lightest SUSY particle (LSP), the signals are characterized by soft final state objects including low missing transverse momentum $\left(\mathbb{E}_{T}\right)$ [1-13]. However, in non-minimal scenarios, the SUSY signals maybe substantially modified in the presence of alternative candidates for LSP and provide valuable probes of detection for the MSSM sector [14, 15]. In such cases, the SUSY signal is characterised by the presence of hard objects and large $\mathbb{E}_{T}$ in the final state. Typical compressed spectra are not restricted to cSUSY scenarios only and also show up in a variety of other new physics scenarios such as extra-dimensions $[16,17]$ as well as in extended gauge sectors [18] demanding further phenomenological studies in this context.

We focus on compressed SUSY scenarios with a higgsino-like $\widetilde{\chi}_{1}^{0}$ (with higgsino fraction $\geq 95 \%$ ) as the next-to-lightest sparticle (NLSP) and a light keV-scale gravitino $(\widetilde{G})$ as the 
LSP and potential dark matter (DM) candidate. We henceforth refer to this scenario as 'constrained' SUSY ( $\mathscr{C}$ SUSY). The rest of the spectrum, comprising of the strong and electroweak sparticles, are compressed in mass with respect to the NLSP. ${ }^{1}$ Such a spectrum has previously been studied in the context of MSSM [14] and its extensions [10, 15] at LHC and Tevatron [19]. In this case, a higgsino-like $\widetilde{\chi}_{1}^{0}$ NLSP decays to a Higgs boson or a $Z$ boson along with the $\widetilde{G}$. Therefore the final states arising from the decay of the heavy sparticles lead to multifarious diboson $(h h, Z Z, Z h)$ signals with large $\mathbb{E}_{T}$.

As the mass scale of new physics extends into the multi-TeV regime new techniques have evolved such as jet substructure techniques [20] have gained importance to study boosted objects. It has been extensively used in various new physics scenarios: vector-like quarks [21], two Higgs doublet models [22, 23], little Higgs [24] and seesaw models [24-26]. The di-Higgs channel along with missing transverse energy $\left(\mathbb{E}_{T}\right)$ is explored in reference [24] using b-tagged jets to reconstruct the Higgs. However, in our current scenario, high $p_{T}$ $b$-jets suffer from low reconstruction efficiency. We study the impact of applying boosted techniques to study the prospects of observing $\mathscr{C}$ SUSY spectra at the $\sqrt{s}=14 \mathrm{TeV}$ run of LHC. We also examine some kinematic observables to distinguish between compressed and uncompressed spectra. The unique points covered in this work are as follows:

- We consider compressed SUSY spectra with a higgsino-like $\widetilde{\chi}_{1}^{0}$ NLSP and a light keV gravitino as the LSP and dark matter candidate. The MSSM sector is compressed within $200 \mathrm{GeV}$ with the NLSP while the NLSP-LSP mass gap is $\mathcal{O}(2 \mathrm{TeV})$. This ensures the presence of a highly boosted Higgs or $Z$ boson in the final state along with $\mathbb{E}_{T}$.

- The boosted Higgs or $Z$ boson are studied in the final state containing at least two fat-jets along with $\mathbb{E}_{T}$. A multivariate analysis is performed using Boosted Decision Tree (BDT) techniques with observables such as N-subjettiness, jet mass and energy correlators used to discriminate between signal and background. The BDT technique shows a clear improvement over conventional cut-based analysis techniques as explicitly demonstrated.

- We also discuss possible new signatures complimentary to the hadronic channel. From a preliminary parton level estimate, we observe that such signatures are more likely to be observable at the proposed high energy and high luminosity upgrade of the LHC at $\sqrt{s}=27 \mathrm{TeV}$. This provides alternate discovery probes to affirm or exclude the presence of a higgsino-like NLSP.

The paper is organised as follows: in section 2 we discuss the relevant decays of the higgsino-like $\widetilde{\chi}_{1}^{0}$ NLSP. In section 3, the current experimental constraints from LHC on the current scenario are discussed and some representative benchmark points satisfying current experimental limits are chosen. The detailed signal and background analysis for the two boosted fat-jets and missing energy is performed and results are presented in section 4 . New kinematic observables to distinguish between compressed and uncompressed spectra are discussed in section 5. Section 6 summarises and concludes the work.

\footnotetext{
${ }^{1}$ For the rest of the paper, we refer to this compression as the compressed spectra.
} 

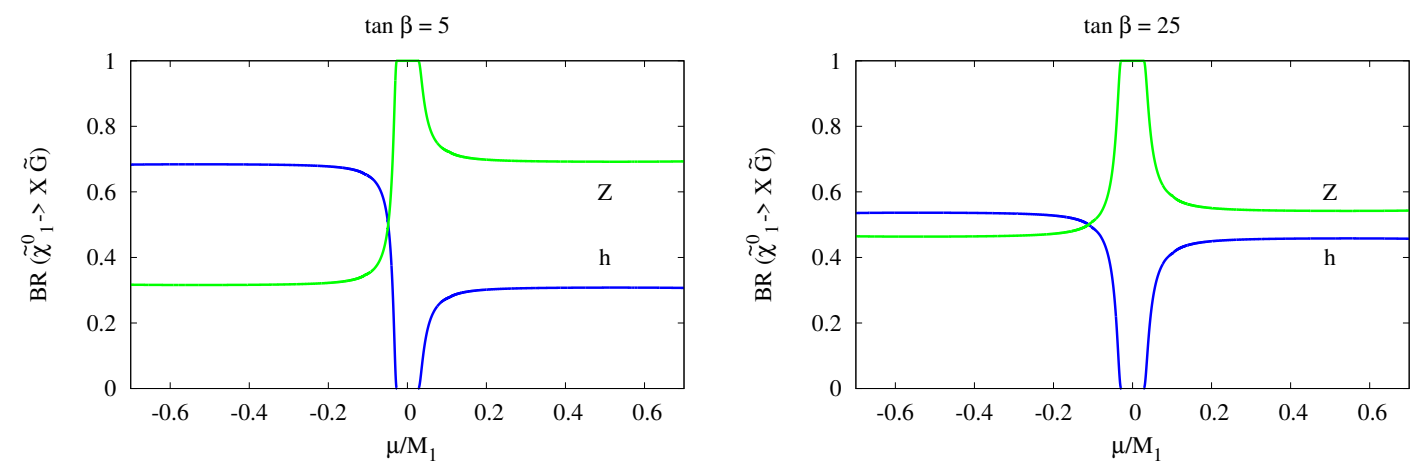

Figure 1. Variation of the branching ratios of the $\widetilde{\chi}_{1}^{0}$ NLSP producing the Higgs (blue lines) or $Z$ boson (green lines) as a function of ratio $\frac{\mu}{M_{1}}$ for fixed values of $M_{1}, M_{2}$. All parameters are shown in table 1 . Two plots are for $\tan \beta=5$ and 25 respectively.

\section{Decay properties of a higgsino-like NLSP}

Our focus is on a compressed MSSM sector with the higgsino-like $\widetilde{\chi}_{1}^{0}$ as the NLSP along with a light $\widetilde{G}$ LSP. For more details we refer the readers to reference [14, 15]. Here we only revisit the relevant decays of the NLSP and the current experimental constraints from LHC that dictate our choice of benchmark points.

The branching ratios of the $\widetilde{\chi}_{1}^{0}$ decay are governed by its composition and therefore on the value of the parameters $M_{1}, M_{2}, \mu$ and $\tan \beta[15,19,27-29]$. For a gaugino-like $\widetilde{\chi}_{1}^{0}$ NLSP, the obvious decay modes to the $Z \widetilde{G}$ and $\gamma \widetilde{G}$ are open whereas for the higgsino-like case, its decay to the Higgs mode $(h \widetilde{G})$ also opens up. The relevant partial decay widths of the lightest neutralino in the decoupling limit $\left(\mu \ll M_{1}, M_{2}\right)$ are [27-29]:

$$
\begin{aligned}
& \Gamma\left(\widetilde{\chi}_{1}^{0} \rightarrow h \widetilde{G}\right) \propto\left|N_{14} \cos \beta+N_{13} \sin \beta\right|^{2}\left(M_{P l} m_{\widetilde{G}}\right)^{-2} \\
& \Gamma\left(\widetilde{\chi}_{1}^{0} \rightarrow Z \widetilde{G}\right) \propto\left(\left|N_{11} \sin \theta_{W}-N_{12} \cos \theta_{W}\right|^{2}+\frac{1}{2}\left|N_{14} \cos \beta-N_{13} \sin \beta\right|^{2}\right)\left(M_{P l} m_{\widetilde{G}}\right)^{-2}
\end{aligned}
$$

where $N_{i j}$ refer to the elements of the neutralino mixing matrix. The terms proportional to $N_{14}$ and $N_{13}$ denote the Goldstone couplings to $h / Z$ and $\widetilde{G}$ whereas $\theta_{W}$ denotes the Weinberg angle and $\tan \beta=v_{u} / v_{d}$ is the ratio of the vev's $v_{u}$ and $v_{d}$ of the two Higgs doublets, $H_{u}$ and $H_{d}$, respectively. Note that for the higgsino-like case there is a huge suppression in branching probability to $\gamma \widetilde{G}$ mode,

$$
\Gamma\left(\widetilde{\chi}_{1}^{0} \rightarrow \gamma \widetilde{G}\right) \propto\left|N_{11} \cos \theta_{W}+N_{12} \sin \theta_{W}\right|^{2}\left(M_{P l} m_{\widetilde{G}}\right)^{-2},
$$

since the photon mode is governed by the bino and wino components which are suppressed as compared to the higgsino fraction.

In figure 1 we plot the variation of the branching ratios of $\widetilde{\chi}_{1}^{0}$ into a Higgs or $Z$ as a function of $\left(\mu / M_{1}\right)$. Corresponding fixed values of $M_{1}, M_{2}$ and other parameters are listed in table 1 where $\mu$ is the higgsino mass parameter, while $M_{1}$ and $M_{2}$ are the bino and wino soft mass parameters respectively. The plots are shown for two values of $\tan \beta=5,25$. We have used SPheno-v3.3.6 $[30,31]$ to scan the parameter space. 


\begin{tabular}{|c|c|c|c|}
\hline Parameters & $|\mu|(\mathrm{TeV})$ & $\operatorname{sign}(\mu)$ & $\tan \beta$ \\
\hline Values & $0.2-2.8$ & \pm 1 & 5,25 \\
\hline
\end{tabular}

Table 1. Relevant range of the input parameters for the parameter-space scan to study the decay probabilities of the lightest neutralino. Other parameters at fixed values which include: $M_{1}=4 \mathrm{TeV}$, $M_{2}=4 \mathrm{TeV}, M_{3}=2.9 \mathrm{TeV}, M_{Q_{3}}=2.8 \mathrm{TeV}, M_{U_{3}}=2.8 \mathrm{TeV}, M_{A}=3.0 \mathrm{TeV}, A_{t}=3.2 \mathrm{TeV}$ and $m_{\widetilde{G}}=1 \mathrm{keV}$.

\begin{tabular}{|c|c|c|c|}
\hline Final state & Production channels & ATLAS & CMS \\
\hline $2 / 3 / 4 b+\mathbb{E}_{T}$ & $\widetilde{\chi}_{1}^{0} \widetilde{\chi}_{1}^{ \pm}, \widetilde{\chi}_{2}^{0} \widetilde{\chi}_{1}^{ \pm}, \widetilde{\chi}_{1}^{+} \widetilde{\chi}_{1}^{-}, \widetilde{\chi}_{1}^{0} \widetilde{\chi}_{2}^{0}$ & {$[32]$} & {$[33]$} \\
$\ell^{+} \ell^{-}+\mathbb{E}_{T}$ & $\widetilde{\chi}_{1}^{0} \widetilde{\chi}_{1}^{ \pm}, \widetilde{\chi}_{2}^{0} \widetilde{\chi}_{1}^{ \pm}, \widetilde{\chi}_{1}^{+} \widetilde{\chi}_{1}^{-}, \widetilde{\chi}_{1}^{0} \widetilde{\chi}_{2}^{0}$ & & {$[33]$} \\
$\geq 3 \ell+\mathbb{E}_{T}$ & $\widetilde{\chi}_{1}^{0} \widetilde{\chi}_{1}^{ \pm}, \widetilde{\chi}_{2}^{0} \widetilde{\chi}_{1}^{ \pm}, \widetilde{\chi}_{1}^{+} \widetilde{\chi}_{1}^{-}, \widetilde{\chi}_{1}^{0} \widetilde{\chi}_{2}^{0}$ & & {$[33]$} \\
$h h+\mathbb{E}_{T}$ & $\widetilde{g} \widetilde{g}$ & & {$[34]$} \\
$4 \ell+\mathbb{E}_{T}$ & $\widetilde{\chi}_{1}^{+} \widetilde{\chi}_{1}^{-}, \widetilde{\chi}_{1}^{ \pm} \widetilde{\chi}_{2}^{0}$ & {$[35]$} & \\
$\geq 2 j+\mathbb{E}_{T}$ & $\widetilde{g} \tilde{g}, \widetilde{q} \widetilde{q}$ & {$[36]$} & {$[37]$} \\
$b \bar{b}+\mathbb{E}_{T}$ & $\widetilde{\chi}_{2}^{0} \widetilde{\chi}_{1}^{ \pm}$ & {$[38]$} & \\
$3 \ell+\mathbb{E}_{T}$ & $\widetilde{\chi}_{2}^{0} \widetilde{\chi}_{1}^{ \pm}$ & {$[38]$} & \\
$\ell^{ \pm} \ell^{ \pm}+\mathbb{E}_{T}$ & $\widetilde{\chi}_{2}^{0} \widetilde{\chi}_{1}^{ \pm}$ & {$[38]$} & \\
$2 b+1 \ell+\mathbb{E}_{T}$ & $\widetilde{\chi}_{1}^{ \pm} \widetilde{\chi}_{2}^{0}$ & {$[39]$} & \\
$\ell^{+} \ell^{-}+\geq 1 j+\mathbb{E}_{T}$ & $\widetilde{\chi}_{1}^{ \pm} \widetilde{\chi}_{2}^{0}, \widetilde{l l}$ & {$[40]$} & \\
\hline
\end{tabular}

Table 2. List of the experimental searches from LHC for higgsinos as relevant for our current study with $\widetilde{G}$ LSP.

We observe a gradual increase of the branching into the Higgs with increasing ratio $\left(|\mu| / M_{1}\right)$ due to an increase of the higgsino fraction in the NLSP. The general features of the plots are summarised below:

- For positive $\left(\mu / M_{1}\right)$, the branching ratios to the $Z \widetilde{G}$ and $h \widetilde{G}$ modes are comparable except in the low $\tan \beta$ regime where the former dominates.

- For negative $\left(\mu / M_{1}\right)$, the $h \widetilde{G}$ decay is greater than $Z \widetilde{G}$ decay, primarily in the low $\tan \beta$ regime.

This motivates choice of regions in the parameter space where either decay mode or both have branching fractions which are substantial in order to explore the multifarious signal possibilites. Accordingly, we choose the representative benchmarks after briefly summarising the relevant experimental constraints in the following section.

\section{Benchmarks}

Before moving on to choose relevant benchmarks for our current study, we list the currently available constraints from LHC in table 2. The current exclusion limits on a light higgsino NLSP and gravitino LSP scenario follow: 
- Stringent limits from ATLAS which arise from searches involving multiple $b$-jets along with missing transverse energy $\left(\mathbb{E}_{T}\right)$ excluding $m_{\widetilde{\chi}_{1}^{0}}<380 \mathrm{GeV}$ for equal branching of the $\widetilde{\chi}_{1}^{0}$ into $h \widetilde{G}$ and $Z \widetilde{G}$ boson. For an increased branching fraction into the Higgs $(100 \%)$, the mass limits strengthen considerably excluding $m_{\widetilde{\chi}_{1}^{0}}<890 \mathrm{GeV}$ [41].

- The CMS Collaboration also sets complementary limits summarized in references [33, $42,43]$. Searches involving multiple $b$-jets and $\mathbb{E}_{T}[43]$ rule out $m_{\widetilde{\chi}_{1}^{0}}<500 \mathrm{GeV}$ for $60 \%$ decay of $\widetilde{\chi}_{1}^{0}$ into $h \widetilde{G}$. A combination of searches involving the hadronic search as well as multiple leptons and diphotons constrain $m_{\widetilde{\chi}_{1}^{0}}$ up to $700 \mathrm{GeV}$ for equal branching of $\widetilde{\chi}_{1}^{0}$ into $h$ and $Z$ along with a $\widetilde{G}$ [33]. The exclusion limit improves slightly for the full decay of the $\widetilde{\chi}_{1}^{0}$ to the Higgs or $Z\left(m_{\widetilde{\chi}_{1}^{0}}<750 \mathrm{GeV}\right)$.

Strongly interacting sparticles are also strongly constrained from LHC searches. A recent study performed using boosted jet techniques in reference [34] studies the final state of at least two fat-jets and $\mathbb{E}_{T}$ excluding gluino masses up to $1.8(2.2) \mathrm{TeV}$ for neutralino LSP mass up to $600 \mathrm{GeV}$ (for $\widetilde{\chi}_{2}^{0}$ decaying into Higgs and/or $Z$ boson). This is a relevant constraint for our current work with $\widetilde{G}$ LSP as the same final state was considered thereby imposing strong constraints on the masses of the coloured sparticles.

We choose benchmark points representative of the parameter space allowed by the LHC for a light higgsino-like NLSP scenario with a keV $\widetilde{G}$ LSP. Our focus is on $\mathscr{C}$ SUSY scenarios as considered in previous studies $[10,14]$ with the lightest higgsino-like $\widetilde{\chi}_{1}^{0}$ as the NLSP. One also has to accommodate constraints from the observation of a light Higgs in the mass range $122-128 \mathrm{GeV}$, constraints from LEP on the sparticles (primarily the lightest chargino) as well as constraints from flavour physics. The details of such contraints are shown in reference [10] for the kind of compressed spectra we are interested in. The presence of the $\widetilde{G}$ relaxes the dark matter (DM) constraints on the MSSM part of the spectrum with a keV $\widetilde{G} \mathrm{DM}$ candidate constituting a warm dark matter candidate [44-48]. We use SPheno-v3.3.6 [30,31] to obtain the benchmarks for the current study. We ensure that the benchmarks chosen pass all the relevant experimental searches from Run 1 and Run 2 at the LHC implemented in CheckMATE-v2.0.26 [49].

Keeping the above constraints in mind, the strongly interacting sector, namely the first and second generation squarks and gluinos, are kept in the mass range $2.4 \mathrm{TeV}-$ $3.0 \mathrm{TeV}$. The third generation squarks are kept heavier than or equal to the first and second generation squarks by choice. In this work we focus on the hadronic signals and choose to keep the electroweak sector heavier than the strong sector. We also focus on a few non-compressed cases to compare the results of our search strategies. Note that our choice of benchmarks are representative of the parameter space involved. The NLSP decaying to the LSP leads to the presence of either Higgs and/or $Z$ bosons in the final state. Thus the expected final states are $h h+\mathbb{E}_{T}, h Z+\mathbb{E}_{T}$ and $Z Z+\mathbb{E}_{T}$, with the light gravitino LSP contributing to the missing transverse momentum $\left(\mathbb{E}_{T}\right)$. The large NLSP-LSP mass gap ensures that the decay products of the NLSP carry high transverse momentum and hence, a large missing energy in the signal as well. The use of jet substructure techniques will thus be very useful to study the hadronic final state products used to reconstruct the 


\begin{tabular}{|c|c|c|c|c|c|c|c|c|}
\hline Parameters & BP1 & BP2 & BP3 & BP4 & BP5 & BP6 & U1 & U2 \\
\hline$M_{1}$ & 2900 & 3000 & 3000 & 3000 & 3500 & 3500 & 2900 & 2900 \\
$\mu$ & 2340 & -2442 & 2505 & 2600 & 2812 & 2910 & 2390 & 1000 \\
$\tan \beta$ & 25 & 25 & 5 & 25 & 25 & 25 & 25 & 25 \\
$A_{t}$ & -3200 & -3200 & -3300 & -3200 & -3200 & -3200 & -3200 & -3200 \\
$m_{A}$ & 2500 & 3000 & 2500 & 2500 & 3000 & 3000 & 3000 & 2500 \\
\hline$m_{h}$ & 124.7 & 124.6 & 122.1 & 124.8 & 124.6 & 124.6 & 124.7 & 124.7 \\
$m_{\widetilde{g}}$ & 2395.1 & 2494.6 & 2609.0 & 2600.9 & 2999.6 & 2953.3 & 3031.7 & 3031.7 \\
$m_{\widetilde{q}_{L}}$ & 2399.1 & 2500.9 & 2603.5 & 2667.7 & 2983.4 & 2961.7 & 2402.1 & 2402.2 \\
$m_{\widetilde{q}_{R}}$ & 2398.0 & 2496.7 & 2599.3 & 2666.4 & 2980.0 & 2960.6 & 2397.8 & 2395.7 \\
$m_{\widetilde{t}_{1}}$ & 2598.5 & 2612.5 & 2638.7 & 2612.5 & 2893.2 & 2929.7 & 2606.4 & 2587.7 \\
$m_{\widetilde{t}_{2}}$ & 2787.5 & 2789.8 & 2845.9 & 2800.2 & 3056.0 & 3096.5 & 2784.7 & 2768.2 \\
$m_{\widetilde{b}_{1}}$ & 2716.1 & 2704.9 & 2734.9 & 2726.6 & 2949.2 & 2985.6 & 2689.2 & 2690.5 \\
$m_{\widetilde{b}_{2}}$ & 2781.3 & 2790.7 & 2789.5 & 2792.3 & 3010.1 & 3047.4 & 2784.7 & 2722.9 \\
$m_{\widetilde{l}_{L}}$ & 3338.3 & 3339.1 & 3339.6 & 3339.1 & 3344.7 & 3345.1 & 3338.1 & 3338.1 \\
$m_{\widetilde{l}_{R}}$ & 3338.5 & 3338.8 & 3338.9 & 3338.8 & 3341.3 & 3341.5 & 3338.4 & 3338.5 \\
$m_{\widetilde{\chi}_{1}^{0}}$ & 2339.5 & 2399.9 & 2498.1 & 2591.0 & 2809.9 & 2905.1 & 1014.2 & 2387.3 \\
$m_{\widetilde{\chi}_{2}^{0}}$ & -2348.7 & -2408.6 & -2510.8 & -2603.4 & -2817.7 & -2914.0 & -1018.1 & -2397.4 \\
$m_{\widetilde{\chi}_{1}^{ \pm}}$ & 2342.7 & 2402.9 & 2502.2 & 2595.1 & 2812.7 & 2908.2 & 1015.9 & 2390.8 \\
$m_{\widetilde{\chi}_{2}^{ \pm}}$ & 2898.6 & 2997.3 & 2997.8 & 3004.1 & 3485.6 & 3486.7 & 2896.2 & 2897.8 \\
$m_{\widetilde{\chi}_{3}^{0}}$ & 2872.5 & 2972.0 & 2971.6 & 2974.4 & 3463.0 & 3462.0 & 2872.5 & 2872.6 \\
$m_{\widetilde{\chi}_{4}^{0}}$ & 2899.0 & 2997.7 & 2998.7 & 3004.8 & 3485.9 & 3487.1 & 2896.2 & 2897.8 \\
\hline$\Delta M_{B R}$ & 59.6 & 101.0 & 110.9 & 76.7 & 189.7 & 56.6 & 2017.5 & 644.4 \\
$B R\left(\widetilde{\chi}_{1}^{0} \rightarrow h \widetilde{G}\right)$ & 0.55 & 0.55 & 0.71 & 0.56 & 0.55 & 0.55 & 0.56 & 0.55 \\
\hline & 0.45 & 0.45 & 0.29 & 0.44 & 0.45 & 0.45 & 0.44 & 0.45 \\
\hline
\end{tabular}

Table 3. List of benchmark points, corresponding parameters and NLSP branching ratios chosen for our study. The mass parameters are in $\mathrm{GeV}$ unless specified otherwise. For all benchmarks, gravitino mass is kept fixed at $m_{\widetilde{G}}=1 \mathrm{keV}$.

boosted $h / Z$ boson in the final states in order to study the $\mathscr{C}$ SUSY spectra. We discuss the analysis techniques and results in section 4 .

We now discuss the salient features of our benchmark points (BP) as listed in table 3. We construct two sets of them as below. While BP1-BP6 represent a compressed spectra with narrow mass difference, $\Delta M<200 \mathrm{GeV}$, U1-U2 are for uncompressed spectra having similar yields.

- BP1-BP6: these represent $\mathscr{C}$ SUSY spectra where one has comparable branching ratio of the $\widetilde{\chi}_{1}^{0} \rightarrow h \widetilde{G}$ and $\widetilde{\chi}_{1}^{0} \rightarrow Z \widetilde{G}$ decay modes. The compression parameter $(\Delta M)$ which is defined as the difference between the mass of the heaviest colored sparticle (i.e, gluinos or the first and second generation squarks) and the NLSP, varies in the range $\Delta M \simeq 56-190 \mathrm{GeV}$ while $m_{\widetilde{\chi}_{1}^{0}} \simeq 2.34-2.91 \mathrm{TeV}$.

- U1-U2: these represent two uncompressed spectra with a lighter $\operatorname{NLSP}\left(m_{\widetilde{\chi}_{1}^{0}} \simeq\right.$ $1.01,2.39 \mathrm{TeV}$ ) with $\Delta M \simeq 2.02,0.64 \mathrm{TeV}$ respectively. 
The different benchmarks involving the compressed spectra vary from one another in the level of mass compression as well as the hierarchical arrangements of the first and second generation squarks and gluinos. For example, BP1-BP3, BP5 and BP6 have a compressed band involving the strong sector sparticles within 5-10 GeV while BP4 accommodates the case where there is a larger mass gap $(\simeq 67 \mathrm{GeV})$ between the squarks and gluinos. This allows the presence of additional light jets in the latter case as compared to the former ones.

\section{Collider analysis}

\subsection{Signal topology}

In the present study, the lightest neutralino has significant higgsino component which opens up new interesting but challenging channels to study. With the above choice, we can have three interesting final states $\left(\tilde{\chi_{1}^{0}} \tilde{\chi_{1}^{0}} \rightarrow h h \tilde{G} \tilde{G}, \tilde{\chi_{1}^{0}} \tilde{\chi_{1}^{0}} \rightarrow Z Z \tilde{G} \tilde{G}, \tilde{\chi_{1}^{0}} \tilde{\chi_{1}^{0}} \rightarrow h Z \tilde{G} \tilde{G}\right)$. It is governed by the benchmarks from table 3 that the Higgs and the $Z$ boson will be highly boosted and the total hadronic activity of the decay of $h / Z$ can be captured in a large radius jet (fat-jet of radius $R$ ), which will be directed by the relation [50]

$$
R \sim \frac{2 M^{h / Z}}{P_{T}^{h / Z}} .
$$

As shown in table 3 the mass of neutralino $\left(\widetilde{\chi}_{1}^{0}\right)$ lies in the range of $2-3 \mathrm{TeV}$. In this case, a Higgs tagger based on b-tagging techniques deteriorates its efficiency [51]. In this process, we also lose a sufficient number of events when $\left(\widetilde{\chi}_{1}^{0}\right)$ is decaying to $Z$ boson. To overcome this issue we propose to capture the Higgs and $Z$ candidate using 2-prong finder tagger which is based on the radiation pattern inside the fat-jet. We utilize the jet substructure techniques to identify $h / Z$ candidate by looking for the following signal topology

$$
p p \rightarrow 2 \text { CA8 Fat-jets }(J)+\text { large } \mathbb{E}_{T},
$$

where CA8 represents the jets clustered with Cambridge-Aachen algorithm with $\mathrm{R}=0.8$. The choice of $\mathrm{R}$ is decided by the relation given in 4.1 such that both the Higgs and $Z$ boson decay products can be captured with the given cut on the fat-jet momentum $\left(P_{T}>300 \mathrm{GeV}\right)$. However, for similar transverse momentum the fat-jets originating from the Higgs boson have a larger radius then the fat-jets originating from the $Z$ boson. Later we utilize 2-prong finder algorithms like N-subjettiness and energy correlation function (ECF) to tag the Higgs or $Z$ like fat-jets.

\subsection{Backgrounds}

The major contribution to the background comes from the following Standard Model processes. Corresponding cross sections as used in present analysis are listed in table 4 with the order of QCD corrections.

- $Z \rightarrow \nu \bar{\nu}+$ jets turns out to be the most dominating background due to large missing transverse momentum and high fake rate of QCD fat-jets as $\mathrm{h} / Z$ jets. 


\begin{tabular}{|c|c|}
\hline Background process & cross section $(\mathrm{pb})$ \\
\hline$Z+$ jets $\left[\mathrm{N}^{2} \mathrm{LO}\right]$ & $6.33 \times 10^{4}[52,53]$ \\
\hline$W+$ jets $[\mathrm{NLO}]$ & $1.95 \times 10^{5}[54]$ \\
\hline Single-top $(t W, t j$ and $t b)\left[\mathrm{N}^{2} \mathrm{LO}\right]$ & $83.1,12.35,248.0[55]$ \\
\hline Diboson $(Z Z, W W, Z W)+$ jets $[\mathrm{NLO}]$ & $17.72,124.31,51.82[56]$ \\
\hline$t \bar{t}+$ jets $\left[\mathrm{N}^{3} \mathrm{LO}\right]$ & $988.57[57]$ \\
\hline
\end{tabular}

Table 4. The cross sections for the background processes used in this analysis are shown with the order of QCD corrections provided in brackets.

- $W \rightarrow l \nu+$ jets contributes to the SM background processes when the lepton is misidentified. Then the dynamics are the same as $Z+$ jets. Due to the large crosssection, these processes contribute significantly.

- $V V+$ jets: diboson production in three different channels, such as, $W_{h} W_{l}, W_{h} Z_{\nu \bar{\nu}}$, and $Z_{h} Z_{\nu \bar{\nu}}$. Here the $V_{h}, V_{l}$ and $V_{\nu \bar{\nu}}$ denotes the hadronic, leptonic and invisible decay modes respectively of $W / Z$ bosons. The diboson process has almost similar signal topology but contributes as a subdominant background due to its low cross-section.

- Single-top production: among the three different productions of the single top $(t W, t j$ and $t b$ ) the main contribution comes from single top associated with $\mathrm{W}$.

- $t \bar{t}$ decaying semi-leptonically has the missing transverse momenta from one of the $W$ decaying leptonically and the possible source for fat-jets is either one of the $\mathrm{W}$ decaying hadronically or mistagged b-jets.

We additionally compute the contributions from the triboson and QCD multijet background which is rendered negligible because of high $\mathbb{E}_{T}$ and two hard fat-jet criteria.

\subsection{Simulated events and data sample}

We have generated the $\mathscr{C}$ SUSY mass spectrum using SPheno-v3.3.6. All the events are generated using Madgraph5 (v2.6.5) [54] at leading order (LO) followed by Pythia (v8) [58] for showering and hadronization. To incorporate detector effects events are passed through Delphes-v3.4.1 [59] using the default CMS card. Delphes tower are used as an input for fat-jet clustering. Fat-jet are reconstructed using the Cambridge-Aachen algorithm [60] with radius parameter $R=0.8$, as implemented in the Fastjet-v3.3.2 [61]. The minimum $p_{T}$ for fat-jet is required to be $300 \mathrm{GeV}$. We use ROOT 5 [62] for the baseline event selection. The final multivariate analysis (MVA) is performed using Boosted Decision Tree (BDT), as implemented in toolkit for Multivariate Analysis TMVA [63]. The events used in the multivariate analysis are selected after the following baseline cuts which are designed for the signal topology discussed in section 4.1.

\section{Baseline selection criteria:}

- We veto the events if any lepton with $p_{T}>10 \mathrm{GeV}$ lies in the central psuedorapidity range $|\eta|<2.4$. 


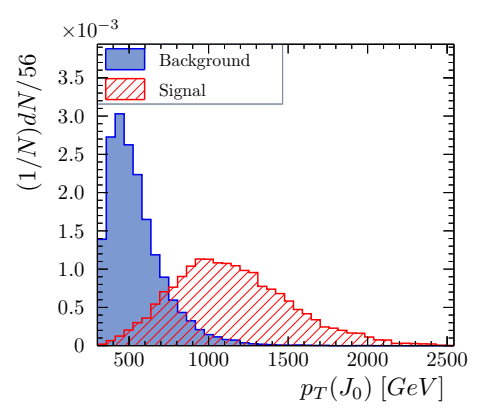

(a)

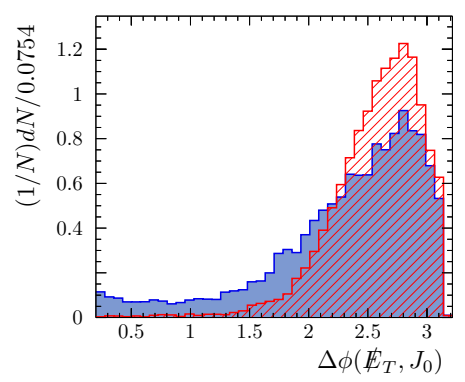

(d)

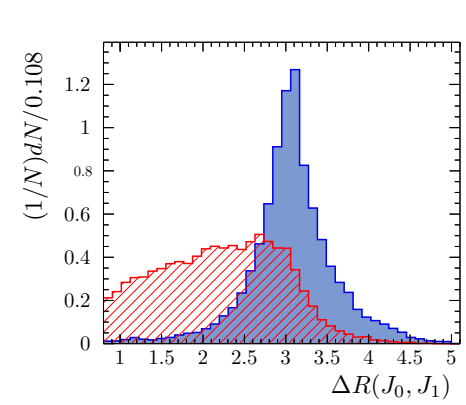

(b)

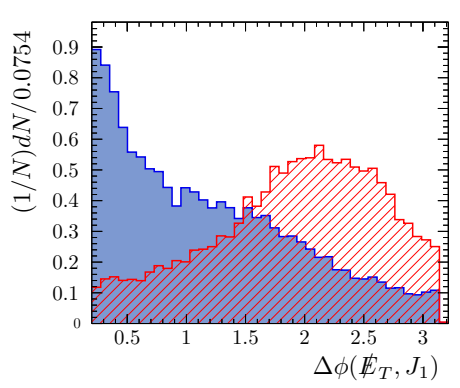

(e)

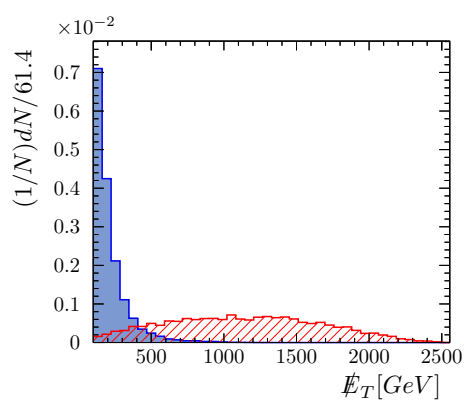

(c)

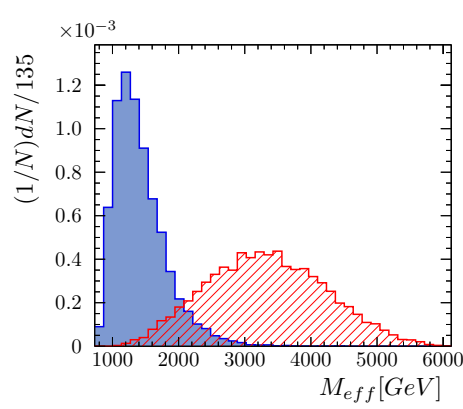

(f)

Figure 2. Normalized distributions of the basic input variables related to two reconstructed fat-jets $J_{i}$ and missing transverse energy $E_{T}^{\prime}$ at the LHC $(\sqrt{s}=14 \mathrm{TeV})$ used in the MVA for the signal (red) and the background (blue). Signal distributions are obtained for benchmark point BP1 and the background includes all the dominant backgrounds.

- We select the events with at least two Cambridge-Aachen fat-jets of radius parameter $=0.8$ and with minimum transverse momentum $p_{T}=300 \mathrm{GeV}$.

- To overcome the effect of jet mismeasurement contributing to missing transverse momenta both the fat-jet should satisfy the criteria of $\left|\Delta \phi\left(J, \mathbb{E}_{T}\right)\right|>0.2$.

- The signal has large missing energy hence we select the events with $E_{T}^{\prime}$ greater than $100 \mathrm{GeV}$.

\subsection{Multivariate analysis}

We perform the collider study using a multivariate analysis (MVA) employing the Boosted Decision Tree (BDT) algorithm. The multivariate analysis outperforms the cut-based analysis since a cut-based analysis can select only one hypercube as the signal region of phase space, whereas, the decision tree can split the phase space into a large number of hypercubes. Each of these hypercubes is then identified as either a 'signal-like' or a 'backgroundlike' tree. Then a non-linear boundary is created in hyperspaces to segregate the signal and background.

We use the following thirteen observables as input to BDT network. The normalized distributions of these input variables are shown in figure 2, figure 3, where the number on Y-axis represents the bin size. 


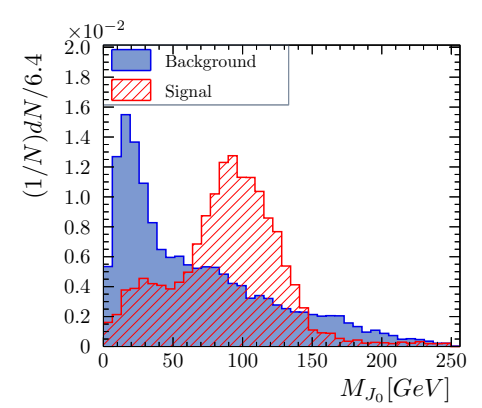

(a)

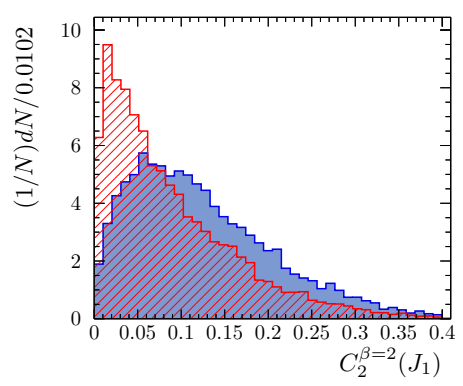

(d)

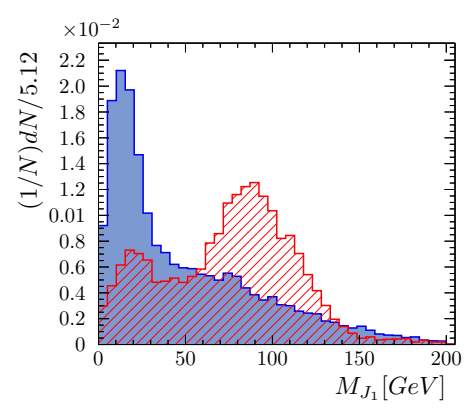

(b)

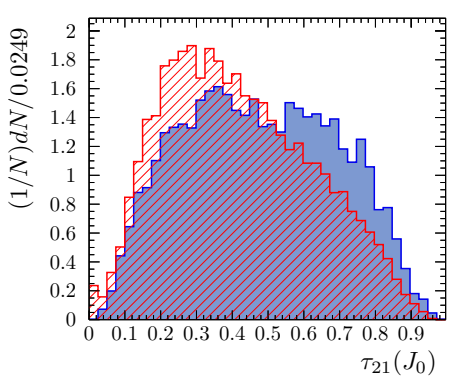

(e)

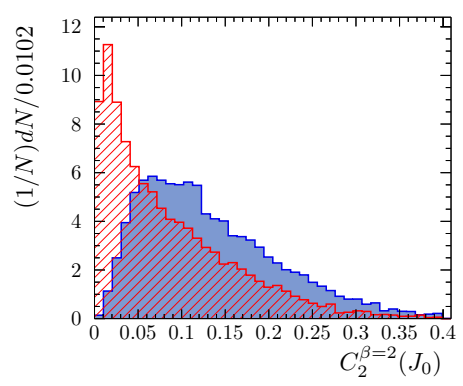

(c)

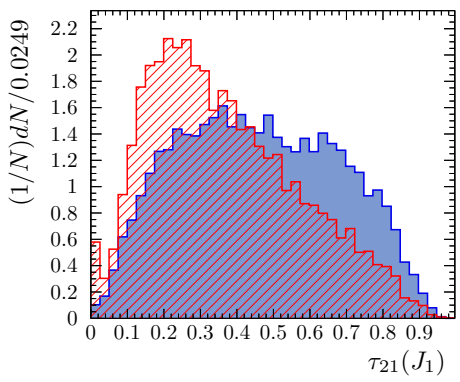

(f)

Figure 3. Normalized distributions of the additional input high level variables constructed for the fat-jets at the LHC $(\sqrt{s}=14 \mathrm{TeV})$ used in the MVA for the signal (red) and the background (blue). Signal distributions are obtained for benchmark point BP1 and the background includes all the dominant backgrounds.

- Transverse momentum of leading fat-jet $P_{T}\left(J_{0}\right)$, figure $2(\mathrm{a})$.

- Transverse momentum of sub-leading fat-jet $P_{T}\left(J_{1}\right)$, similar figure not shown.

- The angular distance difference between two fat-jets $\Delta R\left(J_{0}, J_{1}\right)$, figure 2 (b)

- The missing transverse energy $\mathbb{E}_{T}$, figure 2(c)

- The azimuthal angle difference between missing transverse energy and leading fat-jet $\Delta \phi\left(J_{0}, \mathbb{E}_{T}\right)$, figure $2(\mathrm{~d})$

- The azimuthal angle difference between missing transverse energy and sub-leading fat-jet $\Delta \phi\left(J_{1}, \mathbb{E}_{T}\right)$, figure $2(\mathrm{e})$

- The effective mass of the process $M_{\text {eff }}=\sum_{v i s}\left|P_{T}\right|+\left|\mathbb{E}_{T}\right|$, shown in figure 2(f)

- The mass of leading fat-jet $M_{J_{0}}$ and sub-leading fat-jet $M_{J_{1}}$ are shown in figure 3(a) and figure 3(b), respectively. We used the pruned jet mass by applying the pruning method described in references $[64,65]$ to clean the softer and wide-angle emission. We first calculate $z=\min \left(P_{T i}, P_{T j}\right) / P_{T_{i+j}}$ and the angular separation $\Delta R_{i j}$ between two proto-jets $i$ and $j$ at each step of recombination. Now, the softer proto-jet is discarded if $z<z_{c u t}$ and $\Delta R_{i j}>R_{\text {fact }}$ and $i$-th and $j$-th proto-jets are not 
recombined. Otherwise, $i$-th and $j$-th proto-jets are recombined, and the procedure is repeated unless we remove all the softer and wide-angle proto-jet from the fat-jet. We have used a fixed $R_{\text {fact }}=0.5$ and $z_{c u t}=0.1$ as suggested in reference [64].

- We use 2-prong discriminant energy correlation functions [66]

$$
C_{2}^{(\beta)}=\frac{e_{3}^{(\beta)}}{\left(e_{2}^{(\beta)}\right)^{2}}
$$

where, $e_{2}^{(\beta)}=\sum_{1 \leq i<j \leq n_{J}} z_{i} z_{j} \theta_{i j}^{\beta}$ and $e_{3}^{(\beta)}=\sum_{1 \leq i<j<k \leq n_{J}} z_{i} z_{j} z_{k} \theta_{i j}^{\beta} \theta_{i k}^{\beta} \theta_{j k}^{\beta}$ are 2-point and 3-point energy correlation functions respectively. The $\beta$ represents the exponent. Here $z$ is the energy fraction variable, and $\theta$ is angular variable. The distributions of $C_{2}$ for leading and sub-leading fat-jets are shown in figure 3(c) and 3(d) respectively.

- To reveal the two-prong nature of the fat-jet, we also use the N-subjettiness ratio $[67,68]$

$$
\tau_{N}^{(\beta)}=\frac{1}{\mathcal{N}_{0}} \sum_{i} p_{i, T} \min \left\{\Delta R_{i 1}^{\beta}, \Delta R_{i 2}^{\beta}, \cdots, \Delta R_{i N}^{\beta}\right\}
$$

where, $\mathcal{N}_{0}=\sum_{i} p_{i, T} R_{0}$ is the normalizing factor, $R_{0}$ is the radius parameter of the fat-jet, $\mathrm{N}$ is the axis of the subjet assumed within the fat-jet and $i$ runs over the constituents of the fat-jet. We take the thrust parameter $\beta=2$ which gives more weightage to the angular separation of the constituents from the subjet axis. The distributions of N-subjettiness for leading and sub-leading fat-jets are shown in figure 3(e) and 3(f). We choose One Pass $K_{T}$ Axes for the minimization procedure. The N-subjettiness is slightly less powerful here because of high energetic fat-jets, wherein some events decay products become highly collimated and it is difficult to see a 2-prong structure.

We calculate the linear correlation $\rho$ between two variable $X$ and $Y$ using the following equation

$$
\rho(X, Y)=\frac{E(X Y)-E(X) E(Y)}{\sigma(X) \sigma(Y)}
$$

where $E(X), E(Y)$, and $E(X Y)$ are the expectation value of the variable $X, Y$, and $X Y$ respectively. Here, $\sigma(X) \sigma(Y)$ represents the standard deviation of variable $X$ and $Y$ respectively. Linear correlation among the variables plays a crucial rule to determine the information carried by the variable is unique or not. Most of the variables used in this study are highly uncorrelated with each other as shown in figure 4 . Here positive and negative sign of the coefficients signify correlation and anti-correlation with the other variable. Some set of variables like $\left\{P_{T}\left(J_{0}\right), P_{T}\left(J_{1}\right), M_{\text {eff }}\right\}$ and $\left\{\Delta \Phi\left(E_{T}^{\prime}, J_{0}\right), \Delta \Phi\left(E_{T}^{\prime}, J_{1}\right)\right\}$ show slightly high correlation for signal but have mild correlation in the background. This is mainly because of different kinematics of signal and background processes. Although one should use less correlated variables, some variables with high importance are still used. This is mainly decided when the variable shows a different correlation for the signal and the background. 
Correlation Matrix (background)

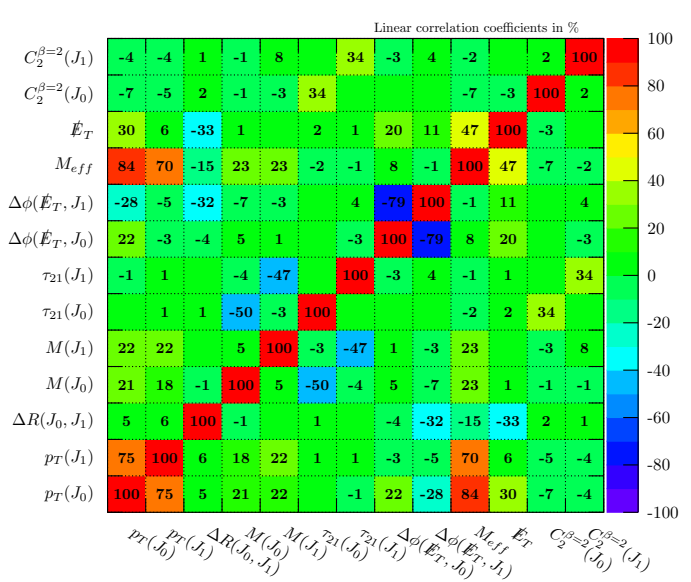

(a)

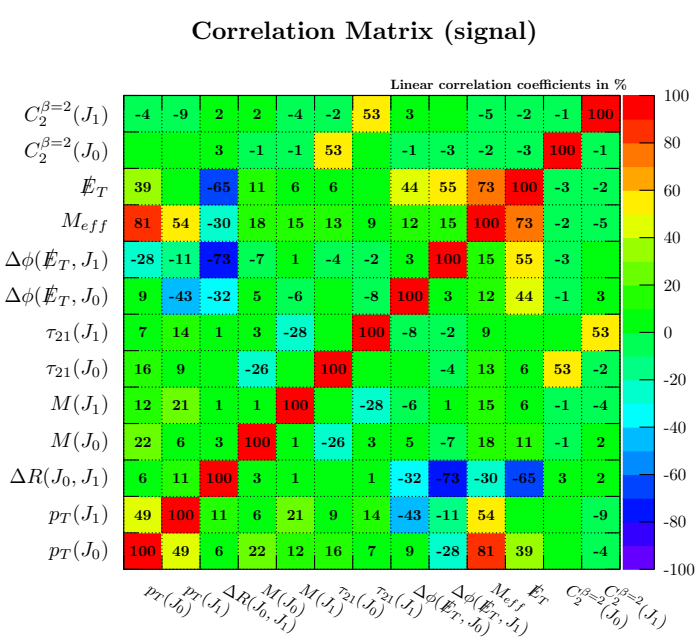

(b)

Figure 4. The linear correlations coefficients (in \%) for (a) signal and (b) background among different kinematical variables that are used for the MVA for benchmark point BP1. Positive and negative signs of the coefficients signify that the two variables are positively correlated and negatively correlated (anti-correlated).

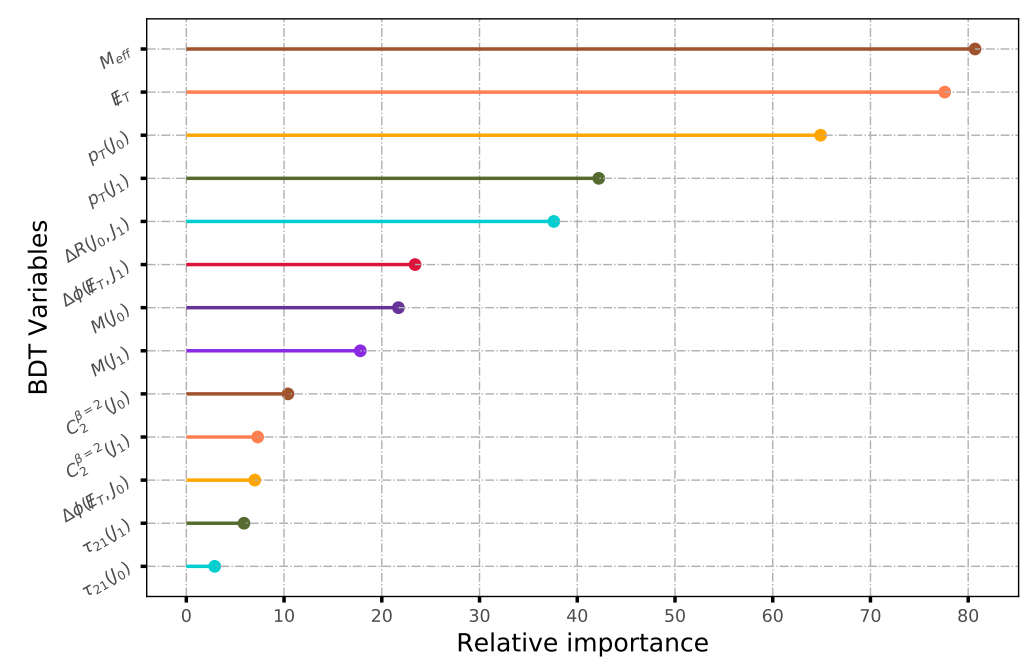

Figure 5. Kinematic variables used for our MVA and their relative importance. We obtain these using numbers from the TMVA package for the benchmark point. Here, we show method unspecific relative importance.

These variables can have less correlation in different regions of phase space after the BDT applies the cuts. If the correlation is different for the signal and the background then a variable is selected/rejected depending on its importance.

We further show the method unspecific ranking (relative importance) for each observable according to their separation in figure 5. The separation in terms of an observable $\lambda$ 


\begin{tabular}{|c|c|c|}
\hline NTrees & 400 & Number of trees in the forest \\
\hline MaxDepth & 2 & Max depth of the decision tree allowed \\
\hline MinNodeSize & $5.6 \%$ & Minimum $\%$ of training events required in a leaf node \\
\hline BoostType & AdaBoost & Boosting type for the trees in the forest \\
\hline AdaBoostBeta & 0.5 & Learning rate for AdaBoost algorithm \\
\hline nCuts & 20 & $\begin{array}{l}\text { Number of grid points in variable } \\
\text { range used in finding optimal cut in node splitting }\end{array}$ \\
\hline
\end{tabular}

Table 5. Parameter used in BDT architecture.

is defined as [63]

$$
\Delta_{(\lambda)}=\int \frac{\left(\hat{y}_{s}(\lambda)-\hat{y}_{b}(\lambda)\right)^{2}}{\hat{y}_{s}(\lambda)+\hat{y}_{b}(\lambda)} d \lambda
$$

where $\hat{y}_{s}$ and $\hat{y}_{b}$ are the probability distribution functions for signal and background for a given observable $\lambda$ respectively. The limits of integration correspond to the allowed range of $\lambda$. Here $\Delta_{(\lambda)}$ quantify discrimination performance of the observable $\lambda$. The separation $\Delta_{(\lambda)}$ ranges from 0 to 1 . If $\Delta_{(\lambda)}=0(0 \%)$ implies $\hat{y}_{s}(\lambda)=\hat{y}_{s}(\lambda)$, which means zero discrimination power of observable $\lambda$ and $\Delta_{(\lambda)}=1(100 \%)$ corresponds to perfect discrimination power.

After calculating the importance of variables, we divide the data set in two equal parts. One part of the data sample is used to train the BDT algorithm and the other part is used for the validation. The parameters used to train the BDT algorithm are shown in table 5 .

Results from BDT analysis considering one sample benchmark point (BP1) is demonstrated in figure 6. Kolmogorov-Smirnov probability for training and testing sample are shown to confirm that the network is not overtrained. The testing data fit well to the training data and the validation is shown in figure 6(a). The BDT is trained for each benchmark point separately. We apply the cut on BDT response and obtain the corresponding number of signal $\mathcal{N}_{S}$ and background $\mathcal{N}_{B}$.

Finally we calculate the statistical significance using formula $\sigma=\mathcal{N}_{S} / \sqrt{\mathcal{N}_{S}+\mathcal{N}_{B}}$. The cut value of $\mathrm{BDT}$ response is $\mathrm{BDT}_{\mathrm{opt}}$, where the maximum significance is achieved. These steps were depicted in second plot for the sample benchmark point, as shown in figure 6(b). Finally, the results for all benchmark points are displayed in table 6 .

\subsection{Complementary signals at high energy and high luminosity upgrades of LHC at $\sqrt{s}=27 \mathrm{TeV}$}

Semi-leptonic and leptonic channels with leptons inside the fat-jet, i.e, lepton-jets are potential alternate channels to confirm the presence of the higgsino-like NLSP besides the hadronic channel. For example, the decay chain $\widetilde{\chi}_{1}^{0} \rightarrow h h / h Z,\left(h \rightarrow W W^{*}\right),(W \rightarrow$ $j j, W \rightarrow l \nu)$ will give rise to an interesting signature of a lepton inside the fat-jet due to high boost of the Higgs. Note that a leptonic decay of the $Z$ boson would also lead to a pair of collimated leptons in the final state. Therefore new signatures with lepton(s) inside jets such as $(j j)(j j),(j j)(l l),(j j)(j j l)$ and $(l l)(l l)$ along with $\mathbb{E}_{T}$ (where $l=e, \mu$ ) may serve as complementary signals to identify the current scenario. We estimate the number of events 


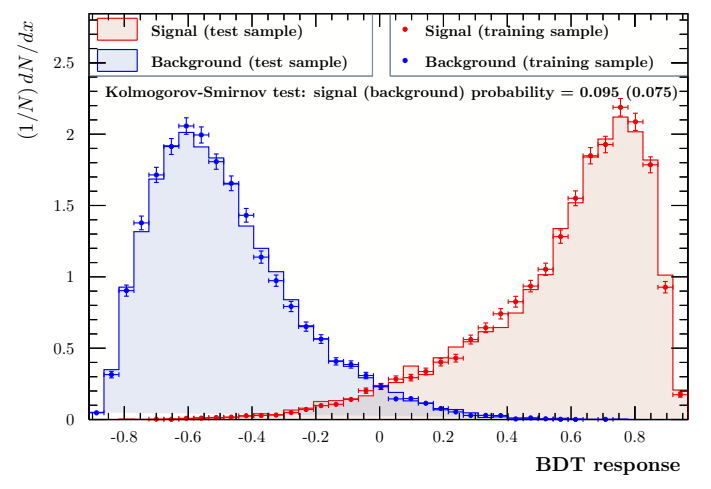

(a)

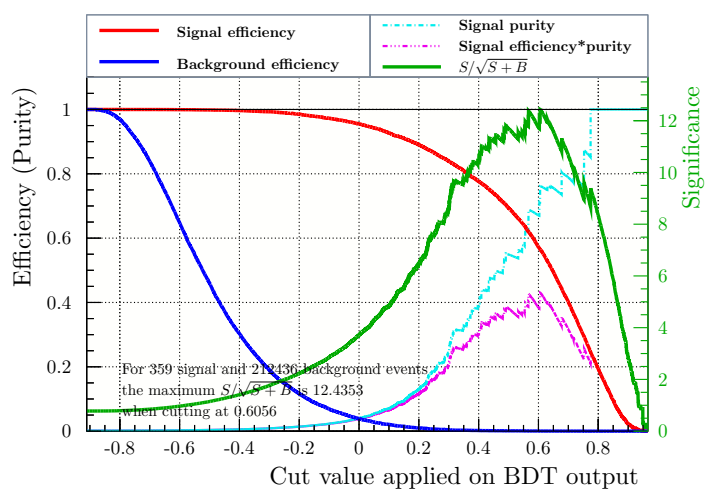

(b)

Figure 6. (a) Normalized BDT response distributions for the signal and the background for the benchmark point BP1. (b) Cut efficiencies as functions of BDT cut values. All plots are evaluated for benchmark point BP1 using integrated luminosity of $200 \mathrm{fb}^{-1}$ at the $14 \mathrm{TeV}$ LHC.

\begin{tabular}{|c|cccccc|}
\hline BPs & $\mathcal{N}_{S}^{b c}$ & BDT $_{\text {opt }}$ & $\mathcal{N}_{S}\left(\epsilon_{S}\right)$ & $\mathcal{N}_{B}\left(\epsilon_{B} \times 10^{4}\right)$ & $\mathcal{N}_{S} / \sqrt{\mathcal{N}_{S}+\mathcal{N}_{B}}$ & $\mathcal{L}_{(5 \sigma)}^{\text {req }} f b^{-1}$ \\
\hline BP1 & 359 & 0.60 & $202(0.56)$ & $63(2.9)$ & 12.4 & 32.3 \\
\hline BP2 & 256 & 0.67 & $137(0.56)$ & $50(2.3)$ & 10.0 & 49.7 \\
\hline BP3 & 346 & 0.42 & $183(0.52)$ & $49(2.3)$ & 12.0 & 34.5 \\
\hline BP4 & 153 & 0.65 & $87(0.56)$ & $15(0.7)$ & 8.6 & 67.4 \\
\hline BP5 & 32 & 0.61 & $25(0.78)$ & $51(2.4)$ & 2.9 & 595.4 \\
\hline BP6 & 74 & 0.58 & $37(0.50)$ & $42(1.9)$ & 4.2 & 283.2 \\
\hline U1 & 266 & 0.57 & $149(0.56)$ & $49(2.3)$ & 10.6 & 44.4 \\
\hline U2 & 352 & 0.56 & $216(0.61)$ & $41(1.9)$ & 13.5 & 27.4 \\
\hline $\mathcal{N}_{\text {SM }}$ & 212436 & - & - & - & - & \\
\hline
\end{tabular}

Table 6. Total number of signal events $\mathcal{N}_{S}^{b c}$ and background events $\mathcal{N}_{\mathrm{SM}}$ before utilising the optimum BDT criteria $\mathrm{BDT}_{\text {opt }}$ for an integrated luminosity of $200 \mathrm{fb}^{-1}$ at the $14 \mathrm{TeV} \mathrm{LHC}$. The number of signal and background events after the BDT ${ }_{\text {opt }}$ cut are denoted by $\mathcal{N}_{S}$ and $\mathcal{N}_{B}$ respectively. Here $\epsilon_{S}$ and $\epsilon_{B}$ represents the signal acceptance and background acceptance efficiency at the $\mathrm{BDT}_{\mathrm{opt}}$ cut value. Finally, listed the statistical significance for an integrated luminosity of $200 \mathrm{fb}^{-1}$ and also required luminosity for a five sigma discovery in case of each BP.

\begin{tabular}{|c|c|c|}
\hline Channel & $\sqrt{s}=14\left(\mathcal{L}=3 a b^{-1}\right) \mathrm{TeV}$ & $\sqrt{s}=27 \mathrm{TeV}\left(\mathcal{L}=15 a b^{-1}\right)$ \\
\hline$(j j)(j j)$ & 4593 & 756177 \\
$(j j)(l l)$ & 352 & 58011 \\
$(l l)(l l)$ & 13 & 2126 \\
$(j j)(j j l)$ & 4 & 664 \\
$(l l)(j j l)$ & 1 & 157 \\
\hline
\end{tabular}

Table 7. Number of events computed using $\sigma * B R$ for BP1 at NLO for $\sqrt{s}=14\left(\mathcal{L}=3 \mathrm{ab}^{-1}\right)$ and $27 \mathrm{TeV}\left(\mathcal{L}=15 \mathrm{ab}^{-1}\right)$ at LHC before analysis cuts are applied. 
prior to signal analysis as summarised in table 7 for $\sqrt{s}=14(27) \mathrm{TeV}$ at $3(15) \mathrm{ab}^{-1}$. We have used the NNPDF [69] parton distribution function to generate the signal events at $\sqrt{s}=27 \mathrm{TeV}$ and obtained the K-factors at NLO from Prospino [70-74].

From table 7 it is observed that the fully hadronic final state $(j j)(j j)$ is the best channel for discovery of the higgsino NLSP scenario over the other leptonic and semi-leptonic channels due to the dominant branching fraction into the hadronic channel. Although the number of events are expected to fall after all detector effects such as reconstruction efficiencies of the jets and leptons are taken into account. Further, signal selection criteria would also lead to reduction in the number of observed events. Therefore, at $\sqrt{s}=14 \mathrm{TeV}$, only the fully hadronic channel is the best possible channel for discovery of the higgsinoNLSP scenario. From section 4 , at $\sqrt{s}=14 \mathrm{TeV}$ we see that the two fat-jet $+\mathbb{E}_{T}$ final state can reach a mass range of $\simeq 2.4 \mathrm{TeV}-3.0 \mathrm{TeV}$ at an integrated luminosity, $\mathcal{L}=200 \mathrm{fb}^{-1}$. Although the semi-leptonic channels $(j j)(l l)$ and $(j j)(j j l)$ can be interesting channels of discovery due to the presence of leptons in the final state, they have relatively fewer events at $\sqrt{s}=14 \mathrm{TeV}$ and are not expected to be significant after detector effects and signal selection efficiencies are taken into account. However such channels would possibly be discoverable at the high energy upgrade of the LHC at $\sqrt{s}=27 \mathrm{TeV}$ as shown in table 7 . The dilepton pair $(l l)$ arising from the decay of the $Z$ boson would also be an indicator of the composition of the NLSP since the $Z$ boson arising from the decay of the higgsinolike NLSP would be longitudinally polarised in the high energy limit where $\sqrt{s} \gg m_{Z}$. On the contrary, a gaugino-like NLSP would give rise to a mostly transversely polarised $Z$ boson. Therefore, the presence of the longitudinal $Z$ boson would be useful to ascertain the higgsino-like nature of the NLSP. Kinematic observables such as $\cos \theta^{*}$ and other variables derived therefrom are useful to explore the polarisation of the $Z$ boson as has been studied in [15] for non-boosted topologies. We leave such studies using boosted techniques for a future work. In addition, channels including a lepton inside a jet, such as $(j j l)$ dominantly arise from the decay of the Higgs, $h \rightarrow W W^{*} \rightarrow j j l$ in the final state. It would be a useful indicator of the presence of a Higgs boson in the final state as opposed to a $Z$ boson and thereby affirming the higgsino-like composition of the NLSP.

\section{Distinction of compressed and uncompressed spectra}

As the results suggest in table 6, the signal yield for different compression is similar for a few benchmarks. It is important to compare the scenario of different compression scale. We define $\Delta M$ as compression scale, where $\Delta M$ is the mass difference between the heaviest colour particle and the NLSP. $\Delta M$ varies from $56-190 \mathrm{GeV}$ for the case of $\mathscr{C}$ SUSY spectra while for uncompressed it is in between $500-2000 \mathrm{GeV}$. With $\widetilde{G}$ being almost massless and NLSP being in the range of $(1-3 \mathrm{TeV})$ we expect that the decay product of NLSP will be sufficiently boosted in both the cases. ${ }^{2}$ Hence both kinds of compression spectra satisfy the loose criteria of at least two fat-jet.

\footnotetext{
${ }^{2}$ Note that direct searches for the weakly interacting NLSP with a gravitino LSP already constrain the mass of such an NLSP to be heavier than $800 \mathrm{GeV}[33,41]$.
} 


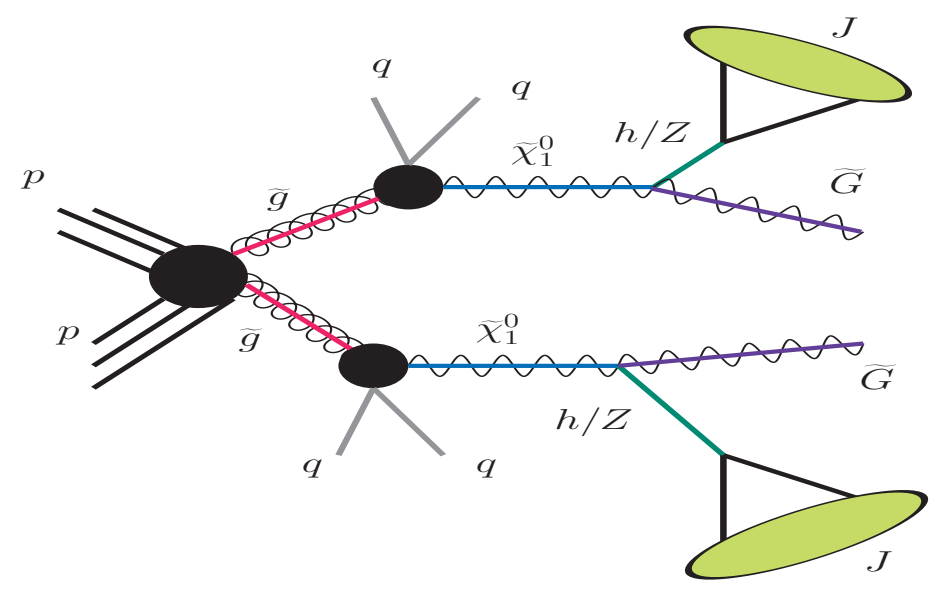

Figure 7. Representative diagram for the signal topology.

A large number of high $p_{T}$ jets are the result of the cascade decay in case of the uncompressed spectrum, whereas the compressed spectrum has very soft jet coming from the cascade decay. Using this information we design two new observables to distinguish these two spectra. To understand the construction of these observables the prototypical signal topology is shown in figure 7 .

We first define the anti-kT jet (AK4) of radius parameter $\mathrm{R}=0.4$ with $P_{T}=20 \mathrm{GeV}$. Further, we identify these AK4 jets $\left(j_{k}\right)$ as "unique jet" jets which are not the part of fat-jet $\left(J_{i}\right)$ i.e. $\Delta R_{J_{i} j_{k}}$ between the reconstructed fat-jet and a AK4 jet is greater than 0.8 hence unique jets are well separated from the fat-jets. ${ }^{3}$ The origin of unique jets is primarily from cascade decay hence they can be identified in a small radius jet.

- The first observable is defined as the ratio of $P_{T}$ of leading unique AK4 jet by the $P_{T}$ of leading fat-jet, written as

$$
\mathcal{Z}_{1}=\frac{P_{T}\left(j_{0}\right)_{\text {unique }}}{P_{T}\left(J_{0}\right)}
$$

- Similarly, we define another variable as the ratio of $P_{T}$ of leading unique jet by the $P_{T}$ of sub-leading fat-jet, written as

$$
\mathcal{Z}_{2}=\frac{P_{T}\left(j_{0}\right)_{\text {unique }}}{P_{T}\left(J_{1}\right)}
$$

The distribution for these variables are shown in figure $8(\mathrm{a})$ and $8(\mathrm{~b})$ respectively. These distributions are plotted with the selected events after the BDT analysis. Evidently, both variables can capture significant information about the compression of the spectrum. The

\footnotetext{
${ }^{3}$ In this analysis, we consider two different classes of jets. AK4 has characteristics and properties such as cone-like regular jet shape which makes it preferable for experimental use, both for jet energy calibration and subtraction of underlying events and pileup. Hence for all small-radius jets, we decided to consider the same. While the CA8 fat-jets are constructed to be used to study the sub-jet structure and variables in the pruning of such jets.
} 


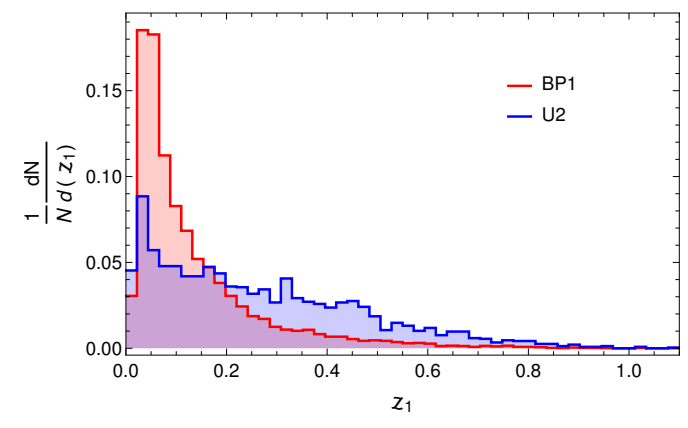

(a)

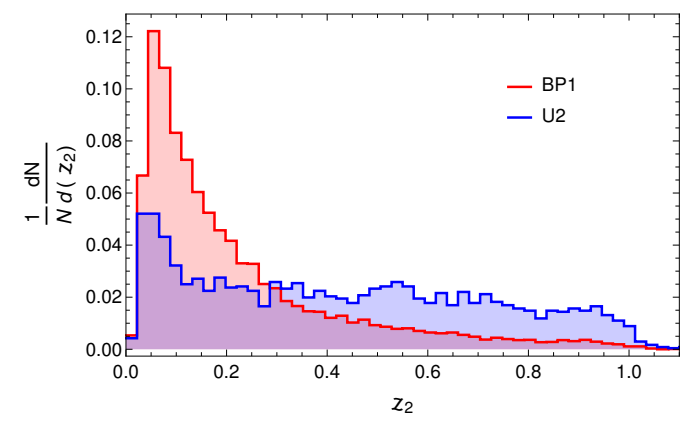

(b)

Figure 8. Normalized distributions of new kinematic variable $\mathcal{Z}_{1}$ and $\mathcal{Z}_{2}$ for the discriminant of compressed and uncompressed spectra.

$\mathcal{Z}_{1}$ and $\mathcal{Z}_{2}$ both have significant contribution at smaller value for BP1 (compressed case) compared to a relatively flat distribution in U2 (uncompressed case). As expected, $p_{T}$ of the leading unique jet is less in case of compressed than in the case of uncompressed spectra and these variables can be used as powerful discriminators in hadronic final state studies of $\mathscr{C}$ SUSY.

\section{Summary and conclusions}

With no clear indication of new physics yet at the LHC, compressed mass spectrum gained significant limelight as a possible explanation for the elusive nature in the realisation of new physics. In this work, we consider a compressed SUSY scenario, where both coloured and electro-weak new physics sectors are sitting at multi-TeV scale in the presence of a light gravitino as dark matter candidate. The lightest neutralino, which is also the natural NLSP candidate in phenomenological MSSM, decays into the gravitino together with Higgs or $Z$-boson. A large mass gap between them invariably produces a significantly boosted boson. Recognising the fact that its hadronic decay can form boosted fat-jet objects opens up an intriguing new possibility. This new channel can be beneficial contrary to looking through the typical leptonic search which is in any case expected to be suppressed by small branching ratio, or reconstruction efficiency at a high $p_{T}$. Moreover, reconstructed fat-jets can still carry the characteristics of the parent particle in their masses and substructures. The present analysis exploits such properties to counter the extensive background coming from QCD jets. With multiple observables, including pruned fat-jet masses, energy correlation functions as well as N-subjettiness, we demonstrate the full potential of jet substructure by using a dedicated multivariate analysis. The LHC sensitivity can be improved substantially that most of the constructed benchmark points can be explored with an integrated luminosity of $200 \mathrm{fb}^{-1}$ at the $14 \mathrm{TeV}$ LHC. One can exclude masses up to $3.2 \mathrm{TeV}$ at $\mathcal{L}=3000 \mathrm{fb}^{-1}$, with a $3.2 \sigma$ signal significance achievable for a compressed spectrum similar to $\mathbf{B P} 6(\Delta M \simeq 60 \mathrm{GeV})$.

At this point, it is worth mentioning that an uncompressed scenario can produce characteristically different signature. We constructed new observables in our present framework 
sensitive to the compression of our model. New possible leptonic and semi-leptonic signatures are also proposed which would be observable at a high energy and high luminosity upgrade of the LHC at $\sqrt{s}=27 \mathrm{TeV}$.

\section{Acknowledgments}

The work of $\mathrm{AB}$ and PK is supported by Physical Research Laboratory (PRL), Department of Space, Government of India and the computations were performed using the HPC resources (Vikram-100 HPC) at PRL. JD, BM and SKR acknowledges support from the Department of Atomic Energy, Government of India, for the Regional Centre for Accelerator-based Particle Physics (RECAPP), Harish-Chandra Research Institute. JD acknowledges support by the Deutsche Forschungsgemeinschaft (DFG, German Research Foundation)under Germany's Excellence Strategy EXC 2121 "Quantum Universe" - 390833306 .

Open Access. This article is distributed under the terms of the Creative Commons Attribution License (CC-BY 4.0), which permits any use, distribution and reproduction in any medium, provided the original author(s) and source are credited.

\section{References}

[1] T.J. LeCompte and S.P. Martin, Large Hadron Collider reach for supersymmetric models with compressed mass spectra, Phys. Rev. D 84 (2011) 015004 [arXiv:1105.4304] [INSPIRE].

[2] T.J. LeCompte and S.P. Martin, Compressed supersymmetry after $1 / \mathrm{fb}$ at the Large Hadron Collider, Phys. Rev. D $8 \mathbf{5}$ (2012) 035023 [arXiv:1111.6897] [INSPIRE].

[3] S.P. Martin, Compressed supersymmetry and natural neutralino dark matter from top squark-mediated annihilation to top quarks, Phys. Rev. D 75 (2007) 115005 [hep-ph/0703097] [INSPIRE].

[4] S.P. Martin, The Top squark-mediated annihilation scenario and direct detection of dark matter in compressed supersymmetry, Phys. Rev. D 76 (2007) 095005 [arXiv:0707.2812] [INSPIRE].

[5] S.P. Martin, Exploring compressed supersymmetry with same-sign top quarks at the Large Hadron Collider, Phys. Rev. D 78 (2008) 055019 [arXiv:0807.2820] [INSPIRE].

[6] H. Dreiner, M. Krämer and J. Tattersall, Exploring QCD uncertainties when setting limits on compressed supersymmetric spectra, Phys. Rev. D 87 (2013) 035006 [arXiv:1211.4981] [INSPIRE].

[7] B. Bhattacherjee and K. Ghosh, Degenerate SUSY search at the $8 \mathrm{TeV} L H C$, arXiv:1207.6289 [INSPIRE].

[8] B. Bhattacherjee, A. Choudhury, K. Ghosh and S. Poddar, Compressed supersymmetry at 14 TeV LHC, Phys. Rev. D 89 (2014) 037702 [arXiv:1308.1526] [InSPIRE].

[9] S. Mukhopadhyay, M.M. Nojiri and T.T. Yanagida, Compressed SUSY search at the $13 \mathrm{TeV}$ LHC using kinematic correlations and structure of ISR jets, JHEP 10 (2014) 012 [arXiv: 1403.6028] [INSPIRE]. 
[10] J. Dutta, P. Konar, S. Mondal, B. Mukhopadhyaya and S.K. Rai, A revisit to a compressed supersymmetric spectrum with 125 GeV Higgs, JHEP 01 (2016) 051 [arXiv: 1511.09284] [INSPIRE].

[11] P. Konar, T. Mondal and A.K. Swain, Demystifying the compressed top squark region with kinematic variables, Phys. Rev. D 96 (2017) 095011 [arXiv: 1612.03269] [INSPIRE].

[12] P. Konar, T. Mondal and A.K. Swain, Constraining slepton and chargino through compressed top squark search, JHEP 04 (2018) 024 [arXiv:1710.08664] [INSPIRE].

[13] N. Nagata, H. Otono and S. Shirai, Cornering compressed gluino at the LHC, JHEP 03 (2017) 025 [arXiv: 1701.07664] [INSPIRE].

[14] J. Dutta, P. Konar, S. Mondal, B. Mukhopadhyaya and S.K. Rai, Search for a compressed supersymmetric spectrum with a light gravitino, JHEP 09 (2017) 026 [arXiv:1704.04617] [INSPIRE].

[15] J. Dutta, B. Mukhopadhyaya and S.K. Rai, Identifying a Higgsino-like NLSP in the context of a keV-scale gravitino LSP, Phys. Rev. D 101 (2020) 075040 [arXiv:1904.08906] [INSPIRE].

[16] S. Dimopoulos, K. Howe, J. March-Russell and J. Scoville, Auto-concealment of supersymmetry in extra dimensions, JHEP 06 (2015) 041 [arXiv:1412.0805] [INSPIRE].

[17] S. Chakraborty, S. Niyogi and K. Sridhar, Constraining compressed versions of MUED and MSSM using soft tracks at the LHC, JHEP 07 (2017) 105 [arXiv: 1704.07048] [INSPIRE].

[18] F.F. Deppisch, N. Desai and T.E. Gonzalo, Compressed and split spectra in minimal SUSY SO(10), Front. in Phys. 2 (2014) 27 [arXiv:1403.2312] [INSPIRE].

[19] K.T. Matchev and S.D. Thomas, Higgs and $Z$ boson signatures of supersymmetry, Phys. Rev. D 62 (2000) 077702 [hep-ph/9908482] [INSPIRE].

[20] J.M. Butterworth, A.R. Davison, M. Rubin and G.P. Salam, Jet substructure as a new Higgs search channel at the LHC, Phys. Rev. Lett. 100 (2008) 242001 [arXiv:0802.2470] [INSPIRE].

[21] CMS collaboration, Search for electroweak production of a vector-like quark decaying to a top quark and a Higgs boson using boosted topologies in fully hadronic final states, JHEP 04 (2017) 136 [arXiv: 1612.05336] [INSPIRE].

[22] A. Bhardwaj, P. Konar, T. Mandal and S. Sadhukhan, Probing the inert doublet model using jet substructure with a multivariate analysis, Phys. Rev. D 100 (2019) 055040 [arXiv: 1905. 04195] [INSPIRE].

[23] R. Patrick, P. Sharma and A.G. Williams, Exploring a heavy charged Higgs using jet substructure in a fully hadronic channel, Nucl. Phys. B 917 (2017) 19 [arXiv:1610.05917] [INSPIRE].

[24] Z. Kang, P. Ko and J. Li, New physics opportunities in the boosted di-Higgs-boson plus missing transverse energy signature, Phys. Rev. Lett. 116 (2016) 131801 [arXiv:1504.04128] [INSPIRE].

[25] A. Das, P. Konar and A. Thalapillil, Jet substructure shedding light on heavy Majorana neutrinos at the LHC, JHEP 02 (2018) 083 [arXiv:1709.09712] [INSPIRE].

[26] A. Bhardwaj, A. Das, P. Konar and A. Thalapillil, Looking for minimal inverse seesaw 
scenarios at the LHC with jet substructure techniques, J. Phys. G 47 (2020) 075002

[arXiv: 1801.00797] [INSPIRE].

[27] S.P. Martin, A Supersymmetry primer, hep-ph/9709356 [INSPIRE].

[28] P. Meade, M. Reece and D. Shih, Prompt decays of general neutralino NLSPs at the Tevatron, JHEP 05 (2010) 105 [arXiv:0911.4130] [InSPIRE].

[29] L. Covi, J. Hasenkamp, S. Pokorski and J. Roberts, Gravitino dark matter and general neutralino NLSP, JHEP 11 (2009) 003 [arXiv:0908.3399] [INSPIRE].

[30] W. Porod and F. Staub, SPheno 3.1: extensions including flavour, CP-phases and models beyond the MSSM, Comput. Phys. Commun. 183 (2012) 2458 [arXiv:1104.1573] [INSPIRE].

[31] W. Porod, SPheno, a program for calculating supersymmetric spectra, SUSY particle decays and SUSY particle production at $e^{+} e^{-}$colliders, Comput. Phys. Commun. 153 (2003) 275 [hep-ph/0301101] [INSPIRE].

[32] ATLAS collaboration, Search for pair production of higgsinos in final states with at least three b-tagged jets in $\sqrt{s}=13$ TeV pp collisions using the ATLAS detector, Phys. Rev. D 98 (2018) 092002 [arXiv: 1806. 04030] [INSPIRE].

[33] CMS collaboration, Combined search for electroweak production of charginos and neutralinos in proton-proton collisions at $\sqrt{s}=13 \mathrm{TeV}$, JHEP 03 (2018) 160 [arXiv:1801.03957] [INSPIRE].

[34] CMS collaboration, Search for physics beyond the standard model in events with high-momentum higgs bosons and missing transverse momentum in proton-proton collisions at 13 TeV, Phys. Rev. Lett. 120 (2018) 241801 [arXiv:1712.08501] [INSPIRE].

[35] ATLAS collaboration, Search for supersymmetry in events with four or more leptons in $\sqrt{s}=13$ TeV pp collisions with ATLAS, Phys. Rev. D 98 (2018) 032009 [arXiv: 1804.03602] [INSPIRE].

[36] ATLAS collaboration, Search for squarks and gluinos in final states with jets and missing transverse momentum using $36 \mathrm{fb}^{-1}$ of $\sqrt{\mathrm{s}}=13 \mathrm{TeV}$ pp collision data with the ATLAS detector, Phys. Rev. D 97 (2018) 112001 [arXiv:1712.02332] [InSPIRE].

[37] CMS collaboration, Search for supersymmetry in proton-proton collisions at $13 \mathrm{TeV}$ in final states with jets and missing transverse momentum, CMS-PAS-SUS-19-006 (2019).

[38] ATLAS collaboration, Search for chargino and neutralino production in final states with a Higgs boson and missing transverse momentum at $\sqrt{s}=13 \mathrm{TeV}$ with the ATLAS detector, Phys. Rev. D 100 (2019) 012006 [arXiv:1812.09432] [INSPIRE].

[39] ATLAS collaboration, Search for direct production of electroweakinos in final states with one lepton, missing transverse momentum and a Higgs boson decaying into two b-jets in pp collisions at $\sqrt{s}=13 \mathrm{TeV}$ with the ATLAS detector, ATLAS-CONF-2019-031 (2019).

[40] ATLAS collaboration, Searches for electroweak production of supersymmetric particles with compressed mass spectra in $\sqrt{s}=13$ TeV pp collisions with the ATLAS detector, Phys. Rev. D 101 (2020) 052005 [arXiv:1911.12606] [INSPIRE].

[41] ATLAS collaboration, https://atlas.web.cern.ch/Atlas/GROUPS/PHYSICS/ CombinedSummaryPlots/SUSY/ATLAS_SUSY_EWSummary_higgsino/

ATLAS_SUSY_EWSummary_higgsino.png. 
[42] CMS collaboration, http://cms-results.web.cern.ch/cms-results/publicresults/publications/SUS-17-004/CMS-SUS-17-004_Figure_012.png.

[43] CMS collaboration, Search for Higgsino pair production in pp collisions at $\sqrt{s}=13 \mathrm{TeV}$ in final states with large missing transverse momentum and two Higgs bosons decaying via $H \rightarrow b \bar{b}$, Phys. Rev. D 97 (2018) 032007 [arXiv:1709.04896] [inSPIRE].

[44] A. Arbey, M. Battaglia, L. Covi, J. Hasenkamp and F. Mahmoudi, LHC constraints on gravitino dark matter, Phys. Rev. D 92 (2015) 115008 [arXiv:1505.04595] [INSPIRE].

[45] L. Covi, M. Olechowski, S. Pokorski, K. Turzynski and J.D. Wells, Supersymmetric mass spectra for gravitino dark matter with a high reheating temperature, JHEP 01 (2011) 033 [arXiv: 1009.3801] [INSPIRE].

[46] M. Viel, J. Lesgourgues, M.G. Haehnelt, S. Matarrese and A. Riotto, Constraining warm dark matter candidates including sterile neutrinos and light gravitinos with WMAP and the Lyman-alpha forest, Phys. Rev. D 71 (2005) 063534 [astro-ph/0501562] [INSPIRE].

[47] E.A. Baltz and H. Murayama, Gravitino warm dark matter with entropy production, JHEP 05 (2003) 067 [astro-ph/0108172] [INSPIRE].

[48] A. Boyarsky, J. Lesgourgues, O. Ruchayskiy and M. Viel, Lyman- $\alpha$ constraints on warm and on warm-plus-cold dark matter models, JCAP 05 (2009) 012 [arXiv:0812.0010] [INSPIRE].

[49] D. Dercks, N. Desai, J.S. Kim, K. Rolbiecki, J. Tattersall and T. Weber, CheckMATE 2: from the model to the limit, Comput. Phys. Commun. 221 (2017) 383 [arXiv:1611.09856] [INSPIRE].

[50] J. Shelton, Jet Substructure, in Searching for New Physics at Small and Large Scales, M. Schmaltz and E. Pierpaoli, World Scientific, Singapore (2013) [arXiv:1302.0260] [INSPIRE].

[51] CMS collaboration, Identification of double-b quark jets in boosted event topologies, CMS-PAS-BTV-15-002 (2016).

[52] S. Catani, L. Cieri, G. Ferrera, D. de Florian and M. Grazzini, Vector boson production at hadron colliders: a fully exclusive QCD calculation at NNLO, Phys. Rev. Lett. 103 (2009) 082001 [arXiv: 0903.2120] [INSPIRE].

[53] G. Balossini et al., Combination of electroweak and QCD corrections to single $W$ production at the Fermilab Tevatron and the CERN LHC, JHEP 01 (2010) 013 [arXiv:0907.0276] [INSPIRE].

[54] J. Alwall et al., The automated computation of tree-level and next-to-leading order differential cross sections, and their matching to parton shower simulations, JHEP 07 (2014) 079 [arXiv: 1405.0301] [INSPIRE].

[55] N. Kidonakis, Theoretical results for electroweak-boson and single-top production, PoS DIS2015 (2015) 170 [arXiv: 1506.04072] [INSPIRE].

[56] J.M. Campbell, R. Ellis and C. Williams, Vector boson pair production at the LHC, JHEP 07 (2011) 018 [arXiv: 1105.0020] [INSPIRE].

[57] C. Muselli, M. Bonvini, S. Forte, S. Marzani and G. Ridolfi, Top quark pair production beyond NNLO, JHEP 08 (2015) 076 [arXiv:1505.02006] [INSPIRE].

[58] T. Sjöstrand, S. Mrenna and P.Z. Skands, PYTHIA 6.4 physics and manual, JHEP 05 (2006) 026 [hep-ph/0603175] [INSPIRE]. 
[59] DELPHES 3 collaboration, DELPHES 3, a modular framework for fast simulation of a generic collider experiment, JHEP 02 (2014) 057 [arXiv:1307.6346] [INSPIRE].

[60] Y.L. Dokshitzer, G.D. Leder, S. Moretti and B.R. Webber, Better jet clustering algorithms, JHEP 08 (1997) 001 [hep-ph/9707323] [INSPIRE].

[61] M. Cacciari, G.P. Salam and G. Soyez, FastJet user manual, Eur. Phys. J. C 72 (2012) 1896 [arXiv:1111.6097] [INSPIRE].

[62] R. Brun and F. Rademakers, ROOT: an object oriented data analysis framework, Nucl. Instrum. Meth. A 389 (1997) 81 [INSPIRE].

[63] A. Hocker et al., TMVA — Toolkit for multivariate data analysis, physics/0703039 [INSPIRE].

[64] S.D. Ellis, C.K. Vermilion and J.R. Walsh, Techniques for improved heavy particle searches with jet substructure, Phys. Rev. D 80 (2009) 051501 [arXiv:0903.5081] [InSPIRE].

[65] S.D. Ellis, C.K. Vermilion and J.R. Walsh, Recombination algorithms and jet substructure: pruning as a tool for heavy particle searches, Phys. Rev. D 81 (2010) 094023 [arXiv:0912.0033] [INSPIRE].

[66] A.J. Larkoski, I. Moult and D. Neill, Power counting to better jet observables, JHEP 12 (2014) 009 [arXiv:1409.6298] [INSPIRE].

[67] J. Thaler and K. Van Tilburg, Identifying boosted objects with N-subjettiness, JHEP 03 (2011) 015 [arXiv: 1011.2268] [INSPIRE].

[68] J. Thaler and K. Van Tilburg, Maximizing boosted top identification by minimizing N-subjettiness, JHEP 02 (2012) 093 [arXiv:1108.2701] [INSPIRE].

[69] R.D. Ball et al., Parton distributions with LHC data, Nucl. Phys. B 867 (2013) 244 [arXiv: 1207.1303] [INSPIRE].

[70] W. Beenakker, R. Hopker and M. Spira, PROSPINO: a program for the production of supersymmetric particles in next-to-leading order $Q C D$, hep-ph/9611232 [INSPIRE].

[71] T. Plehn, Measuring the MSSM Lagrangean, Czech. J. Phys. 55 (2005) B213 [hep-ph/0410063] [INSPIRE].

[72] M. Spira, Higgs and SUSY particle production at hadron colliders, in the proceedings of the $10^{\text {th }}$ International Conference on Supersymmetry and Unification of Fundamental Interactions (SUSY02), June 17-23, Hamburg, Germany (2002), hep-ph/0211145 [INSPIRE].

[73] W. Beenakker, M. Krämer, T. Plehn, M. Spira and P.M. Zerwas, Stop production at hadron colliders, Nucl. Phys. B 515 (1998) 3 [hep-ph/9710451] [InSPIRE].

[74] W. Beenakker, R. Hopker, M. Spira and P.M. Zerwas, Squark and gluino production at hadron colliders, Nucl. Phys. B 492 (1997) 51 [hep-ph/9610490] [InSPIRE]. 